\title{
Ectodermal dysplasia-blindness syndrome
}

INSERM

\section{Source}

INSERM. (1999). Orphanet: an online rare disease and orphan drug data base.

Ectodermal dysplasia-blindness syndrome. ORPHA:1806

Ectodermal dysplasia-blindness syndrome is characterized by intellectual deficit, blindness caused by ocular malformations (microphthalmia, microcornea and sclerocornea), short stature, dysmorphic facial features (narrow nasal bridge and prominent ears),

hypotrichosis, and malaligned teeth. It has been described in two siblings (brother and sister) and is likely to be transmitted as an autosomal recessive trait. 